\title{
Experimental determination of the statistics of photons emitted by a tunnel junction
}

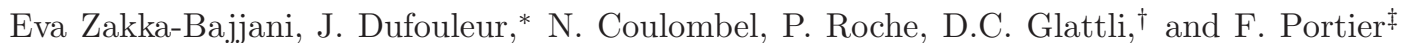 \\ Service de Physique de l'Etat Condensé/IRAMIS/DSM (CNRS URA 2464), CEA Saclay, F-91191 Gif-sur-Yvette, France
}

(Dated: June 4, 2022)

\begin{abstract}
We report on a microwave Hanbury-Brown Twiss experiment probing the statistics of GHz photons emitted by a tunnel junction in the shot noise regime at low temperature. By measuring the crosscorrelated fluctuations of the occupation numbers of the photon modes of both detection branches we show that, while the statistics of electrons is Poissonian, the photons obey chaotic statistics. This is observed even for low photon occupation number when the voltage across the junction is close to $h \nu / e$.

PACS numbers: 73.23.-b,73.50.Td,42.50.-p,42.50.Ar
\end{abstract}

What is the statistics of microwave photons radiated by the current fluctuations of a quantum conductor? When the conductor is at equilibrium at temperature $T$, the statistics is that of a black body radiation [1]. However little is known about the statistics of the photons emitted in the non-equilibrium case where the conductor is biased by a voltage $V \gg k_{B} T / e$ and the current fluctuations are due to quantum shot noise. An intriguing question is the link between the statistics of electrons and that of the emitted photons. This problem has recently attracted theoretical interest and the full range of photon statistics, from chaotic to non-classical, has been predicted. The result depends on the competition between the fermionic and bosonic statistics of electrons and photons respectively [2]. On the one hand, the Pauli principle makes electrons emitted by a contact essentially noiseless and the current noise only results from electron scattering with sub-Poissonian statistics. On the other hand photons emitted by electrons may show bunching effect transforming their initial statistics from sub-Poissonian to super- Poissonian. More generally, this rich physics relates to the problem of the electron Full Counting Statistics [3], as the second moment of the photon noise directly links to a fourth-moment of the current fluctuations [2, 4, 13].

In this work, we present the first measurements of the statistics of photons radiated by a quantum conductor in the shot noise regime. For the simplest quantum conductor studied here, a tunnel junction, although the statistics of electrons crossing the conductor is Poissonian, the photon statistics is shown to be chaotic. This is found even in the regime of vanishing electron shot noise where the voltage is close to the photon energy $(e V \gtrsim h \nu)$, so that the photon population is small, in agreement with the prediction of [2]. As a by-product, our experimental method based on Hanbury-Brown Twiss (HBT) microwave photon correlation is found to provide a direct measurement of the non symmetrized current noise power, called emission noise. Here, the experiments linearly amplifies the field amplitude to a classical level and further detects the microwave power and its fluctuations. This contrasts with experiments measuring the average power with on-chip quantum detectors such as quantum dots or superconducting tunnel junctions [5].

The relation between current noise and photon emission can be understood following Nyquist's approach [1]. Consider a conductor of resistance $R$ connected to a circuit made of a lossless transmission line of characteristic impedance $Z_{c}$ terminated by a matched resistor. To simplify, let's assume that $Z_{c} \ll R$. When electrons in the conductor generate a current fluctuation $I(t)$, a voltage $V(t)=Z_{c} I(t)$ builds up at the input of the transmission line exciting an electromagnetic mode which propagates and is finally absorbed in the resistive load. Introducing the spectral density of the current fluctuation $S_{I}(\nu)=2 \int_{-\infty}^{+\infty}\langle I(0) I(\tau)\rangle e^{i 2 \pi \nu \tau} d \tau$, we can express the electromagnetic power radiated by the conductor in frequency range $\nu, \nu+d \nu$ as $d P=Z_{c} S_{I}(\nu) d \nu=N(\nu) h \nu d \nu$, where $N(\nu)$ is the mean photon population of the electromagnetic mode at frequency $\nu$. This establishes a direct link between $S_{I}(\nu)$ and $N(\nu)$. Let us take a further step and consider the (low frequency) fluctuations $\delta N^{2}$ of the photon population. They originate from the intrinsic fluctuations of the current noise in the conductor (the 'noise' of the noise). However the problem of the connection between the statistics of the photons and the electrons is complicated by the bunching effect occurring when several photons are simultaneously emitted into the transmission line. The photon distribution emitted by a classical current was first addressed by Glauber who showed that the photon statistics is Poissonian [6]. Solving the same problem in the case of quantum electronic shot noise requires a model that treats electrons and the detecting environment on the same quantum footing. Such a treatment was recently developed by Schomerus and Beenakker [2]. In particular they have shown that a tunnel junction emits photons with chaotic statistics. This occurs even in the regime of small photon number when the applied voltage on the junction is close to $h \nu / e$. This is the regime addressed by our experiments.

The experimental set-up, shown in figure 1, is similar to the one described in [7]. An $\mathrm{Al} / \mathrm{AlO}_{x} / \mathrm{Al}$ tunnel junction of resistance $R_{\mathrm{t}}=502 \Omega \pm 1 \Omega$ was cooled to $\sim 30$ $\mathrm{MA}$ in a dilution fridge. A $0.1 \mathrm{~T}$ magnetic field sup- 


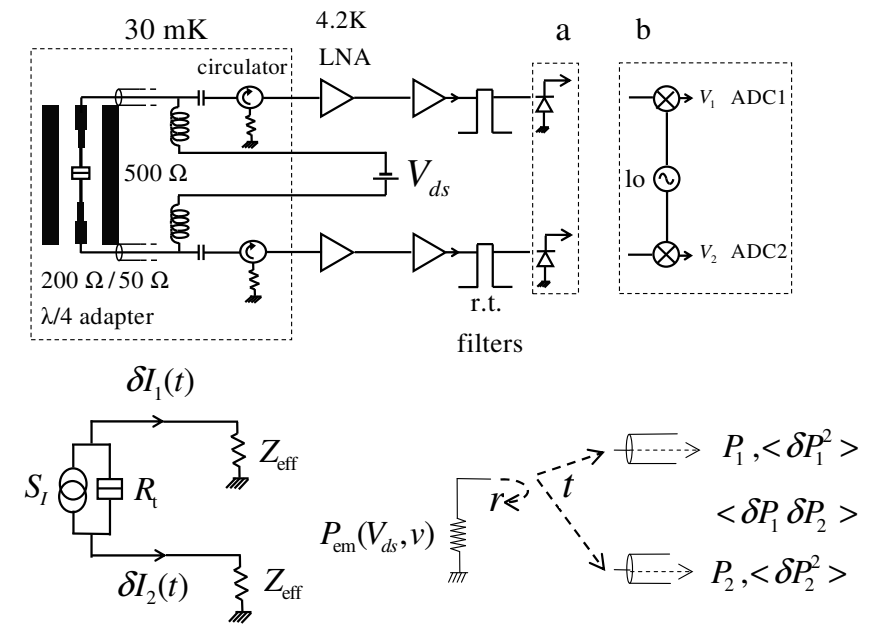

FIG. 1: Schematic diagram of the measurement set-up corresponding to a) detection of the amplified microwave power by quadratic detectors b) fast digitization of the down-converted current fluctuations. c) Equivalent microwave circuit.

presses the $\mathrm{Al}$ superconductivity. The two sides of the tunnel junction are separately connected to $50 \Omega$ coaxial transmission lines via two quarter wave length impedance adapters, raising the effective input impedance of the detection lines to $Z_{\text {eff }}=200 \Omega$ over a one octave bandwidth centered at $6 \mathrm{GHz}$. Two rf-circulators, thermalized at mixing chamber temperature ensure a circuit environment at base temperature.

We note $\delta I_{1,2}$ the fluctuating currents in either detection branches resulting from the fluctuations of the current through the tunnel junction. $S_{I_{1}}, S_{I_{2}}, S_{I_{1}, I_{2}}$ stand for the autocorrelated and cross-correlated spectral densities. From the equivalent circuit represented in figure [1. one easily sees that:

$$
\begin{gathered}
S_{I_{1}}\left(\nu, T, V_{d s}\right)=S_{I_{2}}\left(\nu, T, V_{d s}\right)=-S_{I_{1} I_{2}}\left(\nu, T, V_{d s}\right) \\
=\left(\frac{R_{\mathrm{t}}}{2 Z_{\mathrm{eff}}+R_{\mathrm{t}}}\right)^{2} S_{I}\left(\nu, T, V_{d s}\right),
\end{gathered}
$$

where the two first equalities result from current conservation. The noise power detected in each detection line in a frequency range $\Delta \nu$ reads

$$
P_{1,2}=Z_{\mathrm{eff}} \overline{\delta I_{1,2}^{2}}=\frac{4 Z_{\mathrm{eff}} R_{\mathrm{t}}}{\left(2 Z_{\mathrm{eff}}+R_{\mathrm{t}}\right)^{2}} P_{\mathrm{em}}
$$

where $P_{\mathrm{em}}=R_{\mathrm{t}} S_{I}\left(\nu, T, V_{d s}\right) \Delta \nu / 4$ is the emitted power. This can be expressed as an excess noise temperature $\Delta T_{n 1,2}=P_{1,2} /\left[k_{\mathrm{B}} \Delta \nu_{1,2}\right]$.

The two emitted signals are then amplified by two cryogenic Low Noise Amplifiers. Up to a calculable gain factor, the detected noise power contains the weak excess noise $\Delta T_{n 1,2}$ on top of a large additional noise generated by the cryogenic amplifiers $T_{n 1,2} \simeq 5 \mathrm{~K}$. After fur- ther room temperature amplification and eventually narrow bandpass filtering, current fluctuations are detected using three alternative techniques. First (fig. 1.a), we implemented the measurement scheme described in [7], using two calibrated quadratic detectors whose output voltage is proportional to noise power. Secondly (fig. 1.b), current fluctuations are digitized, after down conversion achieved by mixing with a suited local microwave signal, using an AP240 Acqiris Acquisition Card able to sample at 1 Gsample/s. A quantitative comparison with the well established first method has qualified this new method. The third method, dedicated to the study of photon noise, is a hybridization of the two previous ones: the outputs of the two quadratic detectors are digitized to perform the photon HBT cross and auto correlations fluctuations of $P_{1,2}$.

First experiment: mean photon occupation number. We measure the increase in noise temperature due do the photon emission by shot noise, as a function of $V_{d s}$ and the measuring frequency $\nu$, using the quadratic detectors. In order to remove the background noise of the amplifiers, we measure the excess noise, $\Delta S_{I_{1,2}}\left(\nu, T, V_{d s}\right)=$ $S_{I_{1,2}}\left(\nu, T, V_{d s}\right)-S_{I_{1,2}}(\nu, T, 0)$. Practically, this is done by applying a $93 \mathrm{~Hz} 0-V_{d s}$ square-wave bias voltage on the sample through the DC input of a bias-Tee, and detecting the first harmonic of the square-wave noise response of the detectors using lock-in techniques. The results are quite similar to the one reported in ref. [7], and lead to an electron temperature $T_{e} \sim 70 \mathrm{MA}$. Although $T_{e}$ is significantly higher than the mixing chamber temperature, it is low enough to make the thermal population of photons negligible in the $4-8 \mathrm{GHz}$ frequency range, where all our measurements are done. In the high bias limit $\left(e V_{d s} \gg k_{\mathrm{B}} T, h \nu\right), \Delta S_{I} \sim 2 e I$. Equation 2 then yields an excess noise temperature $\Delta T_{n 1,2}=e V_{d s} / 4 k_{\mathrm{B}}$ in both detection branches in the case of ideal coupling $R_{\mathrm{t}}=2 Z_{\text {eff }}$. In practice, we get excess noise temperatures $\sim 2 \mathrm{~dB}$ lower than expected from the independently measured attenuation of the various microwave components connecting the sample to both amplifiers. $\mathrm{A} \sim 100 \mathrm{fF}$ capacitance for the junction, shunting part of the microwave signal, accounts for this discrepancy. This value is quite reasonable given the area of our tunnel junction $\left(1.4 \mu \mathrm{m}^{2}\right)$.

Second experiment: auto and cross-correlated electronic noise. We record the current fluctuations with the acquisition card using a 5 ns sampling time, chosen large enough to avoid any correlation between successive points, thus maximizing the effective bandwidth. Here again, we eliminate background noise and parasitic correlation between the two inputs of the acquisition card by measuring excess fluctuations. $\Delta \overline{\delta V_{1}^{2}}$ and $\Delta \overline{\delta V_{2}^{2}}$ are proportional to the excess noise power:

$$
\Delta \overline{\delta V_{1,2}^{2}}=G_{1,2} Z_{0} P_{1,2}=G_{1,2} Z_{0} Z_{\mathrm{eff}} \Delta S_{I_{1,2}} \Delta \nu
$$


where $Z_{0}=50 \Omega$ is the input impedance of the acqu tion card, $\Delta \nu$ is the bandpass of the two filters, center around the same frequency $\nu$, and $G_{1,2}$ stands for 1 gain of chain 1,2 . The benefit of this method is that also gives access to the cross-correlation term:

$$
\Delta \overline{\delta V_{1} \delta V_{2}}=\sqrt{G_{1} G_{2}} Z_{0} Z_{\mathrm{eff}} \int_{\Delta \nu} \cos (2 \pi \nu \tau) \Delta S_{I_{1} I_{2}} d \nu
$$

where $\tau$ is the difference of propagation time of elect magnetic waves between the sample and detectors 1 a 2. One easily gets:

$$
\begin{aligned}
\Delta{\overline{\delta V_{1} \delta V_{2}}}_{\text {norm }} & =\frac{\Delta \overline{\delta V_{1} \delta V_{2}}}{\sqrt{\Delta \overline{\delta V_{1}^{2}} \Delta \overline{\delta V_{2}^{2}}}}=-\operatorname{sinc}(\pi \Delta \nu \tau) \cos (2 \pi \\
& \simeq-\cos (2 \pi \nu \tau) \text { for } \Delta \nu \tau \ll 1 .
\end{aligned}
$$

Equation 4 expresses the anticorrelation of current fluctuations $\delta I_{1}$ and $\delta I_{2}$, modified by the phase difference of the microwave signals induced by $\tau$. This is illustrated in Figure 2 for $\nu=6 \mathrm{GHz}$ with $\Delta \nu=100 \mathrm{MHz}$. Here, $\tau$ is varied using two calibrated phase shifters inserted in both detection lines, around a value $\tau_{0}$ which is a priori not known.

As photons emitted at times differing by more than $\sim 1 / \Delta \nu$ do not show correlations, one needs to minimize $\tau$ before measuring the cross-correlated power fluctuations. As $\Delta \nu \ll \nu$, the most sensitive way to do so is to ensure that $\Delta \overline{\delta V_{1} \delta V_{2}}$ norm is constant for various values of $\nu$. The result of such an adjustment is shown in the inset of figure 2. Although small parasitic microwaves reflections introduce extra modulations, $\Delta \overline{\delta V_{1} \delta V_{2}}$ norm doesn't change sign for $4 \mathrm{GHz} \leq \nu \leq 8 \mathrm{GHz}$. This implies that $\tau \leq 125 \mathrm{ps}$, so that $\Delta \nu \tau \ll 1$ and the delay between the two lines doesn't affect the power correlations.

Third experiment: auto and cross-correlated photon noise. We now adopt the original HBT set-up [8], using quadratic detectors connected to the digitizer. This gives access to $S_{P_{1}^{\text {out }} P_{1}^{\text {out }}}$ and $S_{P_{2}^{\text {out }} P_{2}^{\text {out }}}$, and to the crosscorrelation spectrum $S_{P_{1}^{\text {out }}} P_{2}^{\text {out }}$. In the case of a tunnel junction consisting of many weakly transmitted electronic modes, one would expect [9] the emitted photons to follow a negative binomial distribution, as the emitted power results from the incoherent superposition of a large number of sources (the electronic modes of the tunnel junction). This is confirmed by the rigorous treatment of ref. [2], with corrections corresponding to emission of two photons at the same energy by the same electron. In the case of a low impedance (compared to the resistance quantum $h / e^{2}$ ), these corrections are small and one expects the photons emitted by the shot noise power of a tunnel junction to have the same counting statistics as thermal photons, although their origin is quite different and the frequency dependence of photon occupation number $N(\nu)$ doesn't correspond to a Bose-Einstein

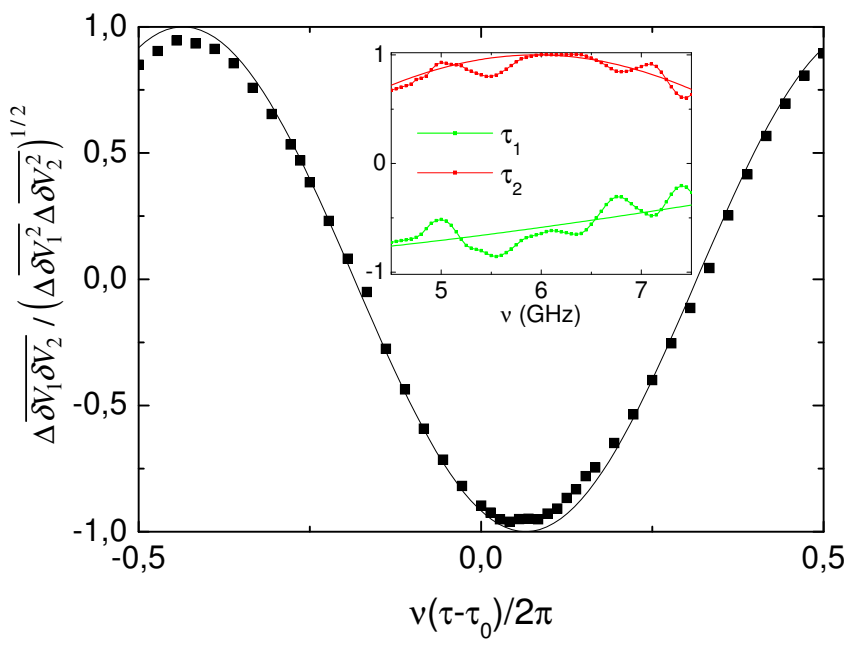

FIG. 2: Normalized cross-correlation spectrum $\Delta \overline{\delta V_{1} \delta V_{2}}$ norm measured at $\nu=6 \mathrm{GHz} \pm 50 \mathrm{MHz}$ as a function of $\nu \Delta \tau$. The solid line represents the sinusoidal prediction of eq. 4 Inset: $\Delta \overline{\delta V_{1} \delta V_{2}}$ norm averaged over $200 \mathrm{MHz}$, as a function of $\nu$ for two values of $\tau$. The solid lines correspond to equation 4 for $\tau=83 \mathrm{ps}$ (upper line) and $\tau=12 \mathrm{ps}$ (lower line).

thermal distribution. The cross-correlated power fluctuations are then expected to be positive, reflecting the bosonic nature of the emitted photons, and proportional to the products of the power emitted in both detection branches. The autocorrelated power fluctuations are expected to be enhanced by the contribution of the noise of the amplifiers 10]. Let's note $\Delta \nu_{\min }\left(\Delta \nu_{\max }\right)$ the smaller (the bigger) of the bandpasses, both centered around the same frequency $\nu$. One thus expects:

$$
\begin{array}{rr}
S_{P_{1}^{\text {out }} P_{1}^{\text {out }}}= & 2 \Delta \nu_{1}\left[G_{1}^{2} k_{\mathrm{B}}^{2}\left(T_{n 1}+\Delta T_{n 1}\right)^{2}+\right. \\
& \left.G_{1} h \nu k_{\mathrm{B}}\left(T_{n 1}+\Delta T_{n 1}\right)\right] \\
\simeq 2 \Delta \nu_{1} G_{1}^{2} k_{\mathrm{B}}^{2}\left(T_{n 1}+\Delta T_{n 1}\right)^{2} \\
S_{P_{1}^{\text {out }} P_{2}^{\text {out }}}=2 G_{1} G_{2} \Delta \nu_{\min } k_{\mathrm{B}}^{2} \Delta T_{n 1} \Delta T_{n 2}
\end{array}
$$

Equation 5 shows the benefit of HBT cross-correlation to study photon statistics, as it suppresses the term due to the mixing of the input power with the input noise of the amplifier. In order to get rid of imperfectly known gains and attenuation we normalize the excess autocorrelated power fluctuations by their zero voltage bias values and the cross-correlated power fluctuations by their geometric mean. Figure 3 represents the excess power fluctuations spectrum $\Delta S_{P_{1}^{\text {out }} P_{1}^{\text {out }}}$, normalized by the zero bias value $S_{P_{1}^{\text {out }} P_{1}^{\text {out }}}\left(V_{d s}=0\right)$ as a function of $\Delta T_{n 1} / T_{n 1}$, measured at $\nu=6.6 \mathrm{GHz} \pm 115 \mathrm{MHZ}$. The solid line represents the theoretical prediction:

$$
\frac{\Delta S_{P_{1}^{\text {out }} P_{1}^{\text {out }}}}{\left[S_{P_{1}^{\text {out }} P_{1}^{\text {out }}}\right]_{V_{d s}=0}}=\left(\frac{\Delta T_{n 1}}{T_{n 1}}\right)^{2}+2 \frac{\Delta T_{n 1}}{T_{n 1}},
$$

which agrees with the experimental observations within $0.5 \%$. Measurements over the entire $4-8 \mathrm{GHz}$ frequency 


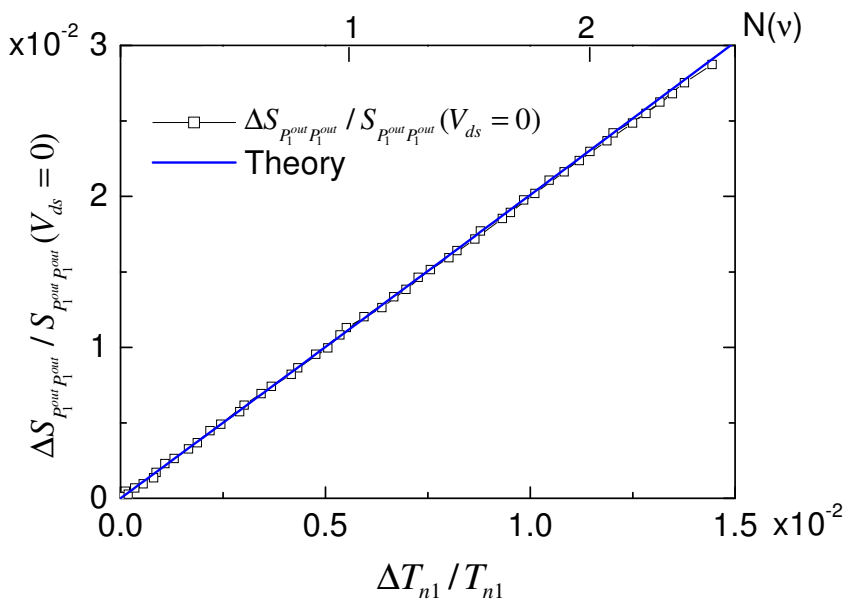

FIG. 3: Normalized auto-correlation spectrum of power fluctuations vs normalized excess noise temperature. Symbols experimental data. Line: Theoretical prediction. The top axis gives the corresponding $N(\nu)$.

range show similar agreement, with a maximum systematic deviation of roughly $3 \%$. As shown by figure 4 the cross-correlated power fluctuations are positive, showing the bosonic character of the emitted excitations. On a quantitative level, one should observe:

$$
\frac{\Delta S_{P_{1}^{\text {out }} P_{2}^{\text {out }}}}{\left[S_{P_{1}^{\text {out }} P_{1}^{\text {out }}} S_{P_{2}^{\text {out }} P_{2}^{\text {out }}}\right]_{V_{d s}=0}^{1 / 2}}=\frac{\Delta T_{n 1}}{T_{n 1}} \frac{\Delta T_{n 2}}{T_{n 2}} \sqrt{\frac{\Delta \nu_{\min }}{\Delta \nu_{\max }}},
$$

Figure 4 shows both the cross-correlated power fluctuations, normalized to the equilibrium auto-correlated power fluctuations, and the product of the excess noise temperatures normalized by $\left(\Delta \nu_{\min } / \Delta \nu_{\max }\right)^{1 / 2}$. They are found to coincide within $4 \%$. As the 'photon noise' is related to a fourth order-correlator of the electronic current [2, 4], this constitutes, to the best of our knowledge, the first measurement of such a correlator in the quantum regime. It shows that the emitted radiation remains chaotic, even when the occupation number of the emitted photon modes tends to zero $\left(e V_{d s}, k_{\mathrm{B}} T \ll h \nu\right)$.

We would like to add a short note to the question of the symmetrization of the current correlator involved in this shot noise measurement. The treatment of Beenakker and Schomerus [2] yields power fluctuations proportional to the square of the electronic emission noise density [12] $S_{I}(\nu)=2 \int_{-\infty}^{+\infty}\langle I(0) I(\tau)\rangle e^{i 2 \pi \nu \tau} d \tau$. Using a symmetrized correlator $S_{I, \text { sym }}=\frac{1}{2}\left(S_{I}(\nu)+S_{I}(-\nu)\right)$ increases the emitted power per unit bandwidth by $h \nu / 2$. As the difference doesn't depend on bias voltage, excess noise measurements cannot distinguish between the two definitions. However, the quadratic dependence of the power fluctuations with the emitted power allows to distinguish them. As shown by figure 4 , assuming power fluctuations $S_{P P} \propto S_{I, \text { sym }}^{2}$ yields a prediction $\Delta S_{P P} \propto$ $\left[\left(\Delta S_{I}\right)^{2}+4 G_{\text {sample }} h \nu \Delta S_{I}\right]$, which is incompatible with

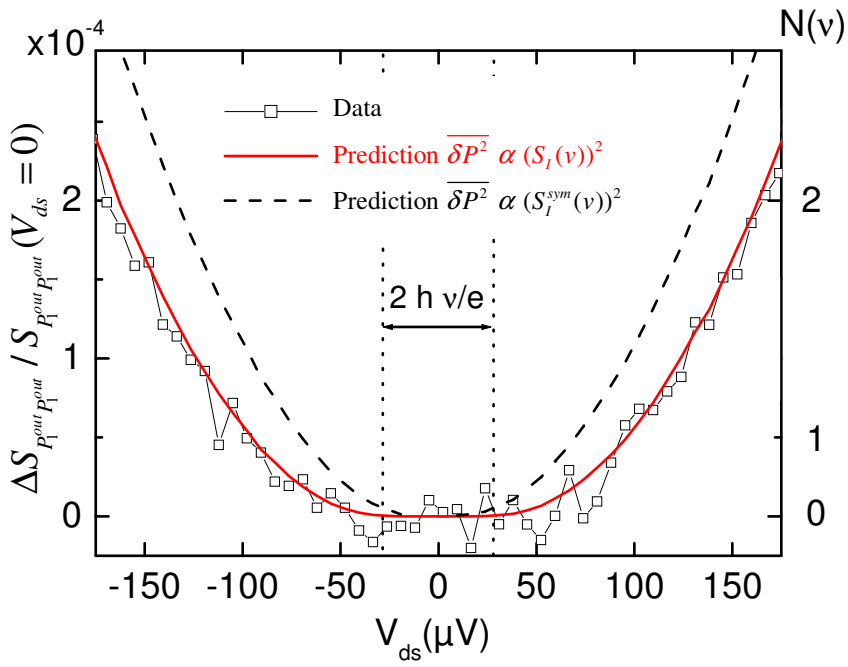

FIG. 4: Open squares: Normalized cross-correlation spectrum of power fluctuations. Solid line: theoretical prediction, assuming $S_{P P} \propto S_{I}^{2}$. Dashed line theoretical prediction, assuming $S_{P P} \propto S_{I, \text { sym }}^{2}$. The right axis gives the corresponding $N(\nu)$. The dotted line represent the onset of finite frequency excess shot noise [5, 7, 11].

our observations.

In conclusion, we have performed the first experiment probing the statistical properties of photons emitted by a phase coherent conductor. The data are found in perfect agreement with the predictions of Beenakker and Schomerus, showing that a biased low impedance tunnel junction emits a chaotic radiation. The crosscorrelated power fluctuations are found to be proportional to the square of the emission electronic noise density. This opens the way to the investigation of the statistical properties of photons emitted by mesoscopic conductors where electronic correlations might have a stronger impact, such as quantum point contacts, for which it is expected that the sub-Poissonian statistics of electronic shot-noise could be 'imprinted' on the corresponding emitted photons [2, 13].

It's a pleasure to acknowledge precious help from Q. Le Masne, P. Bertet and D. Vion with the sample fabrication. We greatly benefited from help during the measurements from B. Dubost. This work was supported by the ANR contract 2e-BQT, and by the C'Nano Idf contract QPC-SinPS.

* Present address: Walter Schottky Institut Technische Universität München Am Coulombwall 3, 85748 Garching, Germany

† Also at Laboratoire Pierre Aigrain, Département de Physique de l'Ecole Normale Supérieure, 24 rue Lhomond, 75231 Paris Cedex 05, France

¥ Electronic address: fabien.portier@cea.fr 
[1] H. Nyquist, Phys. Rev. 32 (1928).

[2] C. W. J. Beenakker and H. Schomerus, Phys. Rev. Lett. 86, 700 (2001); Phys. Rev. Lett. 93, 096801 (2004).

[3] L. Levitov and G. B. Lesovik, JETP Lett. 58, 230 (1993); B. Reulet, J. Senzier, and D. E. Prober, Phys. Rev. Lett. 91, 196601 (2003); S. Gustavsson et al., Phys. Rev. B 75, 075314 (2007); J. Salo, F. W. J. Hekking, and J. P. Pekola, Phys. Rev. B 74, 125427 (2006); Phys. Rev. B 75, 075314 (2007); G. Gershon et al., Phys. Rev. Lett. 101, 016803 (2008); A. Bednorz and W. Belzig, Phys. Rev. Lett. 101, 206803 (2008); J. Gabelli and B.Reulet, J. Stat. Mech. (2008).

[4] J. Gabelli et al., Phys. Rev. Lett. 93, 056801 (2004).

[5] E. Onac et al., Phys. Rev. Lett. 96 (2006); S. Gustavsson et al., Phys. Rev. Lett. 99, 206804 (2007); R. Deblock, E. Onac, and E. G. L. Kouvenhoven, Science 301 (2003).

[6] R. J. Glauber, Phys. Rev. 84, 395 (1951).

[7] E. Zakka-Bajjani et al., Phys. Rev. Lett. 99, 236803 (2007).
[8] R. H. Brown and R. Twiss, Nature 177, 27 (1956).

[9] R. J. Glauber, Phys. Rev. 131, 2766 (1963).

[10] C. M. Caves, Phys. Rev. D 26, 1817 (1982).

[11] G. B. Lesovik, Pis'ma Zh. Éksp. Teor. Fiz. 49, 513 (1997), [JETP Lett. 49, 592 (1989)]; M. Büttiker, Phys. Rev. B 45, 3897 (1992); S.-R.E. Yang, Solid State Commun. 81, 375 (1992); R. Schoelkopf et al., Phys. Rev. Lett. 78, 3370 (1997).

[12] G. B. Lesovik and R. Loosen, Pis'ma Zh. Éksp. Teor. Fiz. 65, 295 (1997), [JETP Lett. 65, 295 (1997)]; G. B. Lesovik, Uspekhi Fiz. Nauk 168, 155 (1998), [PhysicsUspekhi 41, 145 (1998)]; U. Gavish, Y. Levinson, and Y. Imry., Phys. Rev. B 62, R10637 (2000); R. Aguado and L. P. Kouwenhoven, Phys. Rev. Lett. 84, 1986 (2000).

[13] A. V. Lebedev, G. B. Lesovik, and G. Blatter, arXiv:0911.4676v1 (2009). 